HARDY-RAMANUJAN JOURNAL 34 \& 35 (2013), 18-46

\title{
ON RAMACHANDRA'S CONTRIBUTIONS TO TRANSCENDENTAL NUMBER THEORY
}

\author{
Michel WALDSCHMIDT
}

\section{Dedicated to Professor K. Ramachandra on his 70th birthday}

The title of this lecture refers to Ramachandra's paper in Acta Arithmetica [36], which will be our central subject: In section 1 we state his Main Theorem, in section 2 we apply it to algebraically additive functions. Next we give new consequences of Ramachandra's results to density problems; for instance we discuss the following question: let $E$ be an elliptic curve which is defined over the field of algebraic numbers, and let $\Gamma$ be a finitely generated subgroup of algebraic points on $E$; is $\Gamma$ dense in $E(\mathbb{C})$ for the complex topology? The other contributions of Ramachandra to transcendental number theory are dealt with more concisely in section 4 . Finally we propose a few open problems.

The author wishes to convey his best thanks to the organizer of the Madras Conference of July 1993 in honor of Professor Ramachandra's 60th birthday, R. Balasubramanian, for his invitation to participate, which provided him the opportunity to write this paper. Next he is grateful to the organizer of the Bangalore Conference of December 2003 in honor of Professor Ramachandra's 70th birthday, K. Srinivas, for his invitation to participate, which provided him the opportunity to publish this paper. He is also glad to express his deep gratitude to Professor K. Ramachandra for the inspiring role of his work and for his invitation to the Tata Institute as early as 1976.

\section{RAmachandra's Main TheOREM}

Hilbert's seventh problem on the transcendence of $\alpha^{\beta}$ (for algebraic $\alpha$ and $\beta$ ) was solved in 1934 by Gel'fond and Schneider, using two different approaches: while Gel'fond's solution [14] involved the differential equation $(d / d z) e^{z}=e^{z}$ of the exponential function, Schneider's proof [45] rested on the addition formula $e^{z_{1}+z_{2}}=e^{z_{1}} e^{z_{2}}$. Later, both methods were developed and applied to other functions, notably the elliptic functions. In particular Schneider in [46] proved an elliptic analog of the theorem on the transcendence of $\alpha^{\beta}$, using the differential equation which is satisfied by a Weierstrass elliptic function: $\wp^{\prime 2}=4 \wp^{3}-g_{2} \wp-g_{3}$. Sometimes, one refers to Schneider's method when no derivative is needed, and to Gel'fond's 
method when differential equations are there; but, as pointed out by A. Baker, this terminology is somewhat deficient, since for instance Schneider's early results on elliptic functions [46] involve derivatives, and furthermore the first result on functions of several variables (which yields the transcendence of the values of the Beta function at rational points) has been proved by Schneider in [47] using a variant of Gel'fond's method!

The first general criterion dealing with analytic or meromorphic functions of one variable and containing the solution to Hilbert's seventh problem appears in [48]; in fact one can deduce the transcendence of $\alpha^{\beta}$ from this criterion by both methods, either by using the two functions $z$ and $\alpha^{z}$ (Schneider's method), or else $e^{z}$ and $e^{\beta z}$ (Gel'fond's method). This criterion is somewhat complicated, and Schneider made successful attempts to simplify it [49]; however these last results deal only with Gel'fond's method, i.e. derivatives are needed. A further simplification for functions satisfying differential equations was provided by Lang later ([21] and [22]); the socalled Schneider-Lang criterion was used by Bertrand and Masser to derive Baker's Theorem on linear independence of logarithms, as well as its elliptic analog [5]; also it was extended to functions of several variables by Bombieri, solving a Conjecture of Nagata [6].

Thus the situation for functions satisfying differential equations (Gel'fond's method) is rather satisfactory; but it is not the same for Schneider's method. The difficulty of providing simple criteria without assuming differential equations is illustrated by examples due to Weierstrass, Stäckel and others (see [29]). The work of Ramachandra which we consider here deals with this question. Simple criteria are known, the first one being Pólya's Theorem: there is no entire function which is not a polynomial, which maps the natural integers into $\mathbb{Z}$, and which has a growth order less than $2^{z}$ (see [15] Chap. III, §2, for related results, 60] and 63] for surveys, and [59] for a proof which is inspired by Ramachandra's work).

The first part of [36] contains an introduction, the statement of some results and of the Main Theorem, and the proof of it. The second part is devoted to corollaries of the Main Theorem. We reproduce here the Main Theorem.

We denote by $\overline{\mathbb{Q}}$ the field of complex algebraic numbers (algebraic closure of $\mathbb{Q}$ in $\mathbb{C}$ ). The size of an algebraic number $\alpha$ is defined by size $\alpha=\operatorname{den} \alpha+|\alpha|$, where den $\alpha$ is the denominator of $\alpha$ (the least natural integer $d$ such that $d \alpha$ is an algebraic integer) and $\mid \alpha$ is the house of $\alpha$ (maximum of the absolute values of the complex conjugates of $\alpha$ ). We also need the following definition: an entire function $f$ in $\mathbb{C}$ is 
of order $\leq \varrho$ if there exists $C>0$ such that for $R \geq 1$

$$
\log \sup _{|z|=R}|f(z)| \leq C R^{\varrho}
$$

(1) Let $d \geq 2$ be a natural number and $\varrho$ a positive real number; for $1 \leq i \leq d$, let $g_{i}$ and $h_{i}$ be two entire functions without common zeros, of order $\leq \varrho$, and let $M^{(i)}(R)$ denote the quantity

$$
M^{(i)}(R)=\left(1+\max _{|z|=R} \mid h_{i}(z)\right)\left(1+\max _{|z|=R} \mid g_{i}(z)\right) .
$$

Assume further that the $d$ meromorphic functions $f_{i}=h_{i} / g_{i},(1 \leq i \leq d)$ are algebraically independent over $\mathbb{C}$.

(2) Let $\left(\zeta_{\mu}\right)_{\mu \geq 1}$ be an infinite sequence of distinct complex numbers, and $\left(n_{\mu}\right)_{\mu \geq 1}$ be a non-decreasing sequence of natural numbers with $\lim _{\mu \rightarrow \infty} n_{\mu}=\infty$. For $Q \geq 1$, define

$$
N(Q)=\operatorname{Card}\left\{\mu ; \mu \geq 1, n_{\mu} \leq Q\right\} \quad \text { and } \quad D(Q)=\max _{n_{\mu} \leq Q}\left|\zeta_{\mu}\right|,
$$

and assume

$$
\liminf _{Q \rightarrow \infty} \frac{\log N(Q)}{\log D(Q)}>\varrho
$$

(3) Let $\left(\mu_{r}\right)_{r \geq 1}$ be a sequence of integers such that the number

$$
N_{1}(Q)=\operatorname{Card}\left\{\mu_{r} ; r \geq 1, n_{\mu_{r}} \leq Q\right\}
$$

tends to infinity as $Q$ tends to infinity. Suppose that whenever a polynomial in $f_{1}, \ldots, f_{d}$ vanishes at all points $\zeta_{\mu_{r}}$ with $n_{\mu_{r}} \leq Q$, then it vanishes also at all points $\zeta_{\mu}$ with $n_{\mu} \leq Q$

(4) Suppose that the numbers $f_{i}\left(\zeta_{\mu}\right)$ (for $1 \leq i \leq d$ and $\mu \geq 1$ ) are all algebraic numbers; denote by $\partial(Q)$ the degree of the field obtained by adjoining the algebraic numbers

$$
f_{i}\left(\zeta_{\mu}\right), \quad(1 \leq i \leq d, \quad 1 \leq \mu \leq Q)
$$

to the field of rational numbers, and set

$$
M_{1}^{(i)}(Q)=1+\max _{n_{\mu} \leq Q}\left\{\operatorname{size}\left(f_{i}\left(\zeta_{\mu}\right)\right)\right\}, \quad(1 \leq i \leq d)
$$

(5) Finally set

$$
M_{2}^{(i)}(Q)=1+\max _{n_{\mu} \leq Q} \frac{1}{\left|g_{i}\left(\zeta_{\mu}\right)\right|}, \quad(1 \leq i \leq d) .
$$


Main Theorem of [Ramachandra 1968]. Let $q$ be a sufficiently large natural number, and $L_{1}, \ldots, L_{d}$ natural numbers related to $q$ asymptotically by

$$
L_{1} \cdots L_{d} \sim \partial(q)(\partial(q)+1) N_{1}(q) .
$$

Suppose that the hypotheses (1) - (4) above are satisfied. Then there exists a natural number $Q$, greater than $q$, such that for every positive quantity $R$, there holds

$$
1 \leq\left(\frac{8 D(Q)}{R}\right)^{N(Q-1)} \prod_{i=1}^{s}\left(\left(M_{1}^{(i)}(Q)\right)^{8 \partial(Q)} M_{2}^{(i)}(Q) M^{(i)}(R)\right)^{L_{i}}
$$

Remark: It would be interesting to write down a proof of this result by means of Laurent's interpolation determinants (see [24], [25], [26], [27] and [64]): instead of using Dirichlet's box principle (lemma of Thue-Siegel) for constructing an auxiliary function, one considers the matrix of the related system of equations, and one estimates (from below using Liouville's inequality, from above thanks to Schwarz's lemma) a non-vanishing determinant. It is to be expected that such an argument will produce a slightly different explicit inequality, but it is unlikely that these differences will have any effect on the corollaries.

Further works in this direction have been developed; see in particular [57], [30], [59], [16], [17], 34.

The paper [7] can be considered as the first extension of Ramachandra's Theorem to higher dimension; more recent results connected with functions of several variables are given in [62].

\section{Pseudo-Algebraic points of algebraically additive functions}

\section{a) Statement of Ramachandra's upper bound}

When $f$ is a meromorphic function in the complex plane and $y$ is a complex number, we shall say that $y$ is a pseudo-algebraic point of $f$ if either $y$ is a pole of $f$ or else $f(y)$ is an algebraic number (this is the definition in [36] p. 84).

Let $f_{1}, \ldots, f_{d}$ be meromorphic functions; we define $\delta\left(f_{1}, \ldots, f_{d}\right)$ (which is either a non-negative integer or else $\infty$ ) as the dimension of the space of pseudo-algebraic point of $f_{i}$.

This notation is convenient to state a few classical transcendence results: the Theorem of Hermite-Lindemann is $\delta\left(z, e^{z}\right)=0$, the Theorem of Gel'fond-Schneider can be stated either as

-(Gel'fond's method): for any irrational algebraic number $\beta, \delta\left(e^{z}, e^{\beta z}\right)=0$; 
- (Schneider's method): for any non-zero complex number $t, \delta\left(z, e^{t z}\right) \leq 1$.

Schneider's results on elliptic functions (see for instance [49] Chapitre 2 [3 Théorèmes 15, 16 et 18) can also be stated as follows:

if $\wp$ and $\wp^{*}$ are Weierstrass elliptic functions with algebraic invariants $g_{2}, g_{3}, g_{2}^{*}, g_{3}^{*}$, if $\beta$ and $\gamma$ are non-zero algebraic numbers such that the two functions $\wp(z)$ and $\wp^{*}(\gamma z)$ are algebraically independent, if $\zeta$ is the Weierstrass zeta function associated to $\wp$, and if $a, b$ are algebraic numbers with $(a, b) \neq(0,0)$, then

$$
\delta\left(e^{\beta z}, \wp(z)\right)=0, \quad \delta(\wp(z), a z+b \zeta(z))=0
$$

and

$$
\delta\left(\wp(z), \wp^{*}(\gamma z)\right)=0
$$

These results of Schneider on elliptic functions depend heavily on the fact that these functions satisfy differential equations with algebraic coefficients. The main point in Ramachandra's work is that similar results are achieved for functions which do not satisfy differential equations with algebraic coefficients. In place of derivatives, the addition theorem which is satisfied by these functions plays a crucial role. According to 36] p. 85, a meromorphic function $f$ is said to possess an algebraic addition theorem if there exists a non-zero polynomial $P \in \mathbb{C}\left[T_{1}, T_{2}, T_{3}\right]$ such that the meromorphic function of three variables $P\left(f\left(z_{1}+z_{2}\right), f\left(z_{1}\right), f\left(z_{2}\right)\right)$ is the zero function. Further, if there is such a polynomial $P$ with algebraic coefficients, then $f$ will be called algebraically additive.

If $f_{1}, \ldots, f_{d}$ are algebraically additive functions, then the set of common pseudoalgebraic points of $f_{1}, \ldots, f_{d}$ can be shown to be a $\mathbb{Q}$-vector space, and $\delta\left(f_{1}, \ldots, f_{d}\right)$ is nothing else than the dimension of this vector space. The fundamental result in part II of [36] (Theorem 1 p. 74) is the following upper bound for this dimension:

Ramachandra's $\delta$-Theorem. - Let $f_{1}, \ldots, f_{d}$, with $d \geq 2$, be algebraically independent meromorphic functions; assume that for $1 \leq i \leq d$, the function $f_{i}$ is algebraically additive and is of order $\leq \varrho_{i}$. Define

$$
\kappa= \begin{cases}1 & \text { if } f_{1}, \ldots, f_{d} \text { have a common non-zero period } \\ 0 & \text { otherwise }\end{cases}
$$

Then

$$
\delta\left(f_{1}, \ldots, f_{d}\right) \leq \frac{\varrho_{1}+\cdots+\varrho_{d}-\kappa}{d-1}
$$


Lang's criterion for Schneider's method in [22] (Chapter 2, Theorem 2) is the following special case:

For $d=2$ the inequality $\delta\left(f_{1}, f_{2}\right) \leq 2 \varrho^{*}$ holds with $\varrho^{*}=\max \left\{\varrho_{1}, \varrho_{2}\right\}$.

We quote from [36] p. 87: "It may be possible to improve the bound for the dimension given by Theorem 2 probably to 1 in all cases; but even a slight improvement such as $\leq \varrho^{*}+\left(\varrho_{*}-\kappa\right) / d$ appears to be very difficult". (We have substituted $\kappa$ and $d$ to $\theta$ and $s$ respectively to cope with our own notations). The number $\varrho^{*}$ stands for $\max \left\{\varrho_{1}, \ldots, \varrho_{d}\right\}$, while $\varrho_{*}$ stands for $\min \left\{\varrho_{1}, \ldots, \varrho_{d}\right\}$.

It is quite remarkable that no substantial improvement of Ramachandra's $\delta$ Theorem has been obtained after more than a quarter of a century!

We now describe one situation where the assumption of Ramachandra's $\delta$-Theorem are satisfied, and nevertheless the estimate $\delta\left(f_{1}, \ldots, f_{d}\right) \leq 1$ does not hold. Take a Weierstrass elliptic function $\wp$ with algebraic invariants $g_{2}$ and $g_{3}$; let $t$ be a non-zero complex number; consider the two functions $f_{1}(z)=z$ and $f_{2}(z)=\wp(t z)$. From Ramachandra's $\delta$-theorem follows $\delta(z, \wp(t z)) \leq 2$. To start with, assume equality holds: let $\alpha$ and $\beta$ be two $\mathbb{Q}$-linearly independent common pseudo-algebraic points of $f_{1}$ and $f_{2}$; then $u=t \alpha$ and $v=t \beta$ are $\mathbb{Q}$-linearly independent common pseudoalgebraic points of $\wp$, and the quotient $\gamma=u / v=\alpha / \beta$ is algebraic irrational; hence $\delta(\wp(z), \wp(\gamma z)) \geq 1$; from Schneider's above mentioned theorem (1) with $\wp^{*}=\wp$, we deduce that the two functions $\wp(z)$ and $\wp(\gamma z)$ are algebraically dependent; now $\gamma$ is irrational, hence we are in the so-called "CM case": when $\left(\omega_{1}, \omega_{2}\right)$ is a fundamental pair of periods, $\tau=\omega_{2} / \omega_{1}$ is an imaginary quadratic number and the associated elliptic curve has a non trivial ring of endomorphisms.

Conversely, if $\wp$ has complex multiplications, for any $t \in \mathbb{C}^{\times}$the set of common pseudo-algebraic points of the two functions $z$ and $\wp(t z)$ is a $\mathbb{Q}(\tau)$-vector space, and therefore $\delta(z, \wp(t z))$ is even; one example where this vector space has positive dimension is when $t$ is a rational multiple of a period of $\wp$; for instance

$$
\delta\left(z, \wp\left(\omega_{1} z\right)\right)=2 .
$$

We repair Ramachandra's Conjecture as follows:

Ramachandra's $\delta$-Conjecture. - For any non-zero complex number $t$ and any Weierstrass elliptic function $\wp\left(\right.$ resp. $\left.\wp^{*}\right)$ with algebraic invariants $g_{2}, g_{3}$ (resp. $\left.g_{2}^{*}, g_{3}^{*}\right)$, assuming the two functions $\wp(t z)$ and $\wp^{*}(z)$ are algebraically independent,

$$
\delta\left(e^{z}, e^{t z}\right) \leq 1, \quad \delta\left(e^{z}, \wp(t z)\right) \leq 1, \quad \delta\left(\wp(t z), \wp^{*}(z)\right) \leq 1 .
$$


The first inequality is equivalent to the so-called four exponentials Conjecture, which was apparently known to Siegel (see [1]), which was also considered by A. Selberg in the early 40's (personal communication, Hong-Kong, July 1993) and later was proposed by Lang in [22]; an equivalent question is the first of Schneider's eight problems in [49]:

Four exponentials Conjecture. - Let $x_{1}, x_{2}$ be two $\mathbb{Q}$-linearly independent complex numbers, and $y_{1}, y_{2}$ be also two $\mathbb{Q}$-linearly independent complex numbers; then one at least of the four numbers

$$
e^{x_{1} y_{1}}, \quad e^{x_{1} y_{2}}, \quad e^{x_{2} y_{1}}, \quad e^{x_{2} y_{2}}
$$

is transcendental.

A partial result can be proved (which is called the five exponentials Theorem in [62]; the fifth number is $\left.e^{x_{1} / x_{2}}\right)$. A result which is stronger than both the six and the five exponentials Theorems, but which does not include the four exponentials Conjecture, is due to D. Roy [43]). Denote by $\tilde{L}$ the set of linear combinations of logarithms of algebraic numbers, which is the $\overline{\mathbb{Q}}$-vector space spanned by $\{1\} \cup\left\{\ell \in \mathbb{C} ; e^{\ell} \in \overline{\mathbb{Q}}^{\times}\right\}$.

Theorem (D. Roy). - If $x_{1}, x_{2}, x_{3}$ are $\overline{\mathbb{Q}}$-linearly independent complex numbers and $y_{1}, y_{2}$ are $\overline{\mathbb{Q}}$-linearly independent complex numbers, then one at least of the six numbers $x_{i} y_{j}$ is not in $\tilde{L}$.

b) Sketch of proof of Ramachandra's $\delta$-Theorem as a consequence of Ramachandra's Main Theorem

The deduction of the $\delta$-Theorem from the Main Theorem is by no means trivial; Ramachandra had to use Weyl's criterion of equidistribution in order to check the hypotheses concerning the poles of the elliptic functions. Another approach is due to Serre (see lemma 3.4 p. 46 of Waldschmidt, 1973]). Here, in this sketch of proof, we hardly quote problems arising from the poles.

Inside the group of common pseudo-algebraic points of $f_{1}, \ldots, f_{d}$, select a finitely generated subgroup $Y=\mathbb{Z} y_{1}+\cdots+\mathbb{Z} y_{\ell}$ of rank $\ell$. In case $\kappa=1$, choose for $y_{1}$ a common period to $f_{1}, \ldots, f_{d}$. Let $h_{1}, h_{2}, \ldots$ be a numbering of $\mathbb{Z}^{\ell}$, with $h_{\mu}=\left(h_{1 \mu}, \ldots, h_{\ell \mu}\right),(\mu \geq 1)$, such that the sequence

$$
n_{\mu}=\max \left\{\left|h_{1 \mu}\right|, \ldots,\left|h_{\ell \mu}\right|\right\}, \quad(\mu \geq 1)
$$

is non-decreasing, and define the sequence $\zeta_{1}, \zeta_{2}, \ldots$ by

$$
\zeta_{\mu}=h_{1 \mu} y_{1}+\cdots+h_{\ell \mu} \zeta_{\ell}, \quad(\mu \geq 1)
$$


so that

$$
\left\{\zeta_{\mu}, \mu \geq 1\right\}=\left\{h_{1} y_{1}+\cdots+h_{\ell} \zeta_{\ell}, h=\left(h_{1}, \ldots, h_{\ell}\right) \in \mathbb{Z}^{\ell}\right\}=\mathbb{Z} y_{1}+\cdots+\mathbb{Z} y_{\ell} .
$$

In fact the poles of any $f_{i}$ should be removed from this sequence; even more, any point which is too close to a pole should also be omitted, in order to estimate $M_{2}^{(i)}(Q)$; but, as mentioned above, we give here only a sketch of the proof. Suitable positive constants $c_{0}, c_{1}, \ldots$ are then selected, which do not depend on the large integer $q$, so that the Main Theorem can be used with the following inequalities:

$$
\begin{gathered}
\partial(q) \leq c_{0}, \quad M^{(i)}(R) \leq \exp \left(c_{1} R^{\varrho_{i}}\right), \quad(1 \leq i \leq d), \\
c_{2} Q^{\ell} \leq N(Q) \leq c_{3} Q^{\ell}, \quad D(Q) \leq c_{4} Q, \\
\max \left\{M_{1}^{(i)}(Q), M_{2}^{(i)}(Q)\right\} \leq \exp \left(c_{5} Q^{\varrho_{i}}\right), \quad(1 \leq i \leq d) .
\end{gathered}
$$

In the case where $\kappa=1$, we define $\left\{\mu_{1}, \mu_{2}, \ldots\right\}$ as the sequence of integers $\mu$ such that $h_{1 \mu}=0$, and we use the bound

$$
N_{1}(Q) \leq c_{6} Q^{\ell-\kappa}
$$

in the case $\kappa=0$, we define $\mu_{r}=r,(r \geq 1)$, and again we have the same upper bound for $N_{1}(Q)$. Now choose

$$
L_{i}=\left[c_{7} q^{\lambda-\varrho_{i}}\right], \quad(1 \leq i \leq d), \quad \text { with } \quad \lambda=\left(\ell-\kappa+\varrho_{1}+\cdots+\varrho_{d}\right) / d,
$$

and choose also $R=c_{10} Q$, where $c_{10}$ is sufficiently large, so that the desired conclusion follows from the Main Theorem.

Remark: We need to take for $L_{i}$ natural integers; we have introduced an integral part, but we need to check that $L_{i}$ does not vanish; hence the sketch of proof is valid only when

$$
\lambda>\varrho^{*}, \quad \text { where } \varrho^{*}=\max \left\{\varrho_{1}, \ldots, \varrho_{d}\right\} .
$$

This condition can be written

$$
\varrho^{*} \leq \frac{\varrho_{1}+\ldots+\varrho_{d}+\ell-\kappa}{d}
$$

since the goal is to prove the upper bound $\ell \leq\left(\varrho_{1}+\cdots+\varrho_{d}-\kappa\right) /(d-1)$, we may consider that this condition on $\varrho^{*}$ is satisfied as soon as

$$
\varrho^{*} \leq \frac{\varrho_{1}+\ldots+\varrho_{d}-\kappa}{d-1}
$$

this assumption occurs explicitly in Theorem 1 p. 74 of [36]; however it is possible to remove it, by means of an induction argument on $d$; see p. 78 of [57] and p. 52 of [58]. 
The statement of Theorem 1 in [36] also involves a notion of weighted sequences which has been used in [57].

c) Corollaries

We first provide a collection of upper bounds for $\delta\left(f_{1}, \ldots, f_{d}\right)$, where $f_{i}$ are either linear, or exponential, or elliptic functions; all these estimates follow from Ramachandra's $\delta$-Theorem.

We start with Gel'fond-Schneider's Theorem (already quoted above): for any nonzero complex number $t, \delta\left(z, e^{t z}\right) \leq 1$.

Another example which do not involve elliptic functions is the six exponentials Theorem (see below): if $x_{1}, \ldots, x_{d}$ are $\mathbb{Q}$-linearly independent complex numbers with $d \geq 2$, then $\delta\left(e^{x_{1} z}, \ldots, e^{x_{d} z}\right) \leq d /(d-1)$. As a matter of fact it suffices to select either $d=2$ or else $d=3$ to cover all cases (see below).

The next examples all involve elliptic functions. Notations are as follows: $\wp, \wp^{*}, \wp_{1}, \ldots$ are Weierstrass elliptic functions, all of whose invariants $g_{2}, g_{3}, g_{2}^{*}, g_{3}^{*}, \ldots$ are algebraic. The numbers $t, t^{*}, t_{1}, \ldots$ are non-zero complex numbers, while $\omega, \omega^{*}, \omega_{1} \ldots$ are respectively non-zero periods of $\wp, \wp^{*}, \wp_{1}, \ldots$ Then

$$
\begin{array}{clcl}
\delta(z, \wp(z)) & \leq 2, & & \\
\delta\left(e^{z}, \wp(t z)\right) & \leq 3, & \delta\left(e^{2 \pi i z}, \wp(\omega z)\right) & \leq 2, \\
\delta\left(\wp(t z), \wp^{*}(z)\right) & \leq 4, & \delta\left(\wp(\omega z), \wp^{*}\left(\omega^{*} z\right)\right) & \leq 3, \\
\delta\left(e^{z}, \wp(t z), \wp^{*}\left(t^{*} z\right)\right) & \leq 2, & & \\
\delta\left(\wp_{1}\left(t_{1} z\right), \wp_{2}\left(t_{2} z\right), \wp_{3}(z)\right) & \leq 3, & \delta\left(\wp_{1}\left(\omega_{1} z\right), \wp_{2}\left(\omega_{2} z\right), \wp_{3}\left(\omega_{3} z\right)\right) & \leq 2, \\
\delta\left(\wp_{1}\left(t_{1} z\right), \wp_{2}\left(t_{2} z\right), \wp_{3}\left(t_{3} z\right), \wp_{4}(z)\right) & \leq 2 . &
\end{array}
$$

We tacitly assumed that the functions we consider are algebraically independent; by the way, it was a non-trivial problem to provide explicit conditions which guarantee the algebraic independence of the functions; an important contribution to this question is Lemma 7 in [36] p. 83; this problem has been solved later in [8].

We now consider more closely a few of these results.

Example 1. $\delta\left(z, e^{t z}\right)$. - Hilbert's seventh problem by Schneider's method

Corollary 1. - Let $\alpha$ be a non-zero complex algebraic number, and $\beta$ an irrational algebraic number; choose any determination $\log \alpha$ of the logarithm of $\alpha$ with $\log \alpha \neq 0$ in case $\alpha=1$. Then $\alpha^{\beta}=\exp (\beta \log \alpha)$ is a transcendental number.

Proof: This statement (Theorem of Gel'fond-Schneider) follows from Ramachandra $\delta$-Theorem by taking

$$
d=2, \quad f_{1}(z)=z, \quad f_{2}(z)=\alpha^{z}=\exp (z \log \alpha), \quad \varrho_{1}=0, \quad \varrho_{2}=1, \quad \kappa=0 ;
$$


since $y_{1}=1$ is a common pseudo-algebraic point of $f_{1}$ and $f_{2}$, we deduce $\delta\left(f_{1}, f_{2}\right)=1$, and hence $\beta$ is not a pseudo-algebraic point of $f_{2}$, which means that $\alpha^{\beta}$ is a transcendental number.

Notice that Lang's above mentioned criterion (2) for Schneider's method in [22] does not cover the transcendence of $\alpha^{\beta}$ : the point is that the orders of the two functions are 0 and 1 respectively, and it is not sufficient to consider the maximum of both numbers.

Example 2. $\delta\left(e^{z}, e^{t z}\right)$. - The six exponentials Theorem

The story starts with Ramanujan's study of highly composite numbers; see [1], [20], 21], 222, [36], [37]; see also [2], 58] and [64] for further references.

Corollary 2. - Let $x_{1}, \ldots, x_{d}$ be $\mathbb{Q}$-linearly independent complex numbers, and $y_{1}, \ldots, y_{\ell}$ be also $\mathbb{Q}$-linearly independent complex numbers; assume that the $d \ell$ numbers

$$
e^{x_{i} y_{j}}, \quad(1 \leq i \leq d, \quad 1 \leq j \leq \ell)
$$

are all algebraic; then $d \ell \leq d+\ell$.

Proof. Take

$$
f_{i}(z)=e^{x_{i} z} \quad \text { and } \quad \varrho_{i}=1 \quad \text { for } \quad 1 \leq i \leq d
$$

Example 3: $\delta\left(e^{z}, \wp(t z)\right)$

Corollary 3. - Let $\lambda_{1}, \ldots, \lambda_{\ell}$ be complex numbers which are linearly independent over $\mathbb{Q}$ such that the $\ell$ numbers $e^{\lambda_{j}},(j=1, \ldots, \ell)$ are algebraic. Let $\wp$ be a Weierstrass elliptic function with algebraic invariants $g_{2}, g_{3}$, and let $v_{1}, \ldots, v_{\ell}$ be pseudo-algebraic point of $\wp$, not all of which are zero.

a) If $\ell \geq 4$, then the matrix with 2 rows and $\ell$ columns

$$
\left(\begin{array}{lll}
\lambda_{1} & \ldots & \lambda_{\ell} \\
u_{1} & \ldots & u_{\ell}
\end{array}\right)
$$

has rank 2.

b) Assume $\lambda_{1}$ is a rational multiple of $2 \pi i$, and $u_{1}$ is a period of $\wp$; then the same conclusion holds for $\ell=3$.

c) If Ramachandra's $\delta$-Conjecture $\delta\left(e^{z}, \wp(t z)\right) \leq 1$ holds, the same conclusion is valid already for $\ell \geq 2$.

A nice consequence of part b) of this statement is Corollary p. 87 of [36] which we reproduce here: 
If $a$ and $b$ are real positive algebraic numbers different from 1 for which $\log a / \log b$ is irrational and $a<b<a^{-1}$, then one at least of the two numbers

$$
\begin{gathered}
x=\left(\frac{1}{240}+\sum_{n=1}^{\infty} \frac{n^{3} a^{n}}{1-a^{n}}\right) \prod_{n=1}^{\infty}\left(1-a^{n}\right)^{-8}, \\
y=\left\{\frac{6}{\left(b^{1 / 2}-b^{-1 / 2}\right)^{4}}-\frac{1}{\left(b^{1 / 2}-b^{-1 / 2}\right)^{2}}-\sum_{n=1}^{\infty} \frac{n^{3} a^{n}\left(b^{n}+b^{-n}\right)}{1-a^{n}}\right\} \prod_{n=1}^{\infty}\left(1-a^{n}\right)^{-8},
\end{gathered}
$$

is transcendental.

Ramachandra deduces from his results some new transcendental complex numbers, by means of the clean trick (p. 68): if $x$ and $y$ are real numbers, then the complex number $x+i y$ is transcendental if and only if one at least of the two numbers $x, y$ is transcendental. Further consequences of this idea have been worked out by G. Diaz in [11] and [12]. See also 68].

Example 4: $\delta\left(\wp(t z), \wp^{*}(z)\right)$

We consider now two elliptic functions (compare with [36] p. 68).

Corollary 4. - Let $\wp$ and $\wp^{*}$ be two Weierstrass elliptic functions with algebraic invariants $g_{2}, g_{3}$ and $g_{2}^{*}, g_{3}^{*}$ respectively; let $u_{1}, \ldots, u_{\ell}$ be $\mathbb{Q}$-linearly independent complex numbers, each of which is a pseudo-algebraic point of $\wp$; similarly, let $u_{1}^{*}, \ldots, u_{\ell}^{*}$ be pseudo-algebraic points of $\wp^{*}$ which are linearly independent over $\mathbb{Q}$. Assume that the two functions $\wp\left(u_{1} z\right)$ and $\wp^{*}\left(u_{1}^{*} z\right)$ are algebraically independent. a) Assume $\ell \geq 5$. Then the matrix

$$
\left(\begin{array}{lll}
u_{1} & \ldots & u_{\ell} \\
u_{1}^{*} & \ldots & u_{\ell}^{*}
\end{array}\right)
$$

has rank 2.

b) Assume $u_{1}$ is a period of $\wp$ and $u_{1}^{*}$ is a period of $\wp^{*}$; then the rank of the matrix is 2 also when $\ell=4$.

c) If Ramachandra's $\delta$-Conjecture $\delta\left(\wp(t z), \wp^{*}(z)\right) \leq 1$ holds, the same conclusion is true if only $\ell \geq 2$.

An impressive collection of further consequences to Ramachandra's Main Theorem is displayed in [8] section III C. Several of the previous corollaries deal with elliptic integrals of the first of second kind; further consequences concern elliptic integrals of the third kind as well. More generally, a natural situation where all hypotheses are satisfied is connected with analytic subgroups of commutative algebraic groups (see for instance [57] $\S 3$ section 5: "Application du théorème de Ramachandra à l'étude de 
sous-groupes à un paramètre de certaines variétés de groupes"; see also [7] and [62]); however it seems to the author that this is not exactly the right place to develop this aspect of the theory.

\section{Applichtion to Density statements}

Ramachandra's results on algebraic values of algebraically additive functions can be used to prove some density results. We give here a sample of results dealing with $\left(\mathbb{R}^{\times}\right)^{2}, \mathbb{C}^{\times}, \mathbb{R}^{\times} \times E(\mathbb{R}), E(\mathbb{R}) \times E^{*}(\mathbb{R})$ and $E(\mathbb{C})$. Further topological groups are considered in [65].

a1) Consequences of the six exponentials Theorem: real case

Let $\gamma_{1}, \ldots, \gamma_{\ell}$ be multiplicatively independent elements in $\left(\mathbb{R}_{+}^{\times}\right)^{2}$; write

$$
\gamma_{j}=\left(\alpha_{j}, \beta_{j}\right), \quad(j=1, \ldots, \ell) .
$$

By means of a well-known result due to Kronecker 1 , one can show that the subgroup $\Gamma$ which is generated by $\gamma_{1}, \ldots, \gamma_{\ell}$ is dense in $\left(\mathbb{R}_{+}^{\times}\right)^{2}$ if and only if for each $s \in \mathbb{Z}^{\ell} \backslash\{0\}$, the matrix with three rows and $\ell$ columns

$$
\operatorname{det}\left(\begin{array}{ccc}
\log \alpha_{1} & \cdots & \log \alpha_{\ell} \\
\log \beta_{1} & \cdots & \log \beta_{\ell} \\
s_{1} & \cdots & s_{\ell}
\end{array}\right)
$$

has rank 3. An obvious necessary condition is that for all $(a, b) \in \mathbb{Z}^{2} \backslash\{0\}$, at least two of the $\ell$ numbers

$$
a \log \alpha_{1}+b \log \beta_{1}, \ldots, a \log \alpha_{\ell}+b \log \beta_{\ell}
$$

are $\mathbb{Q}$-linearly independent. We assume now that this condition is satisfied, and also that the $2 \ell$ numbers $\alpha_{j}$ and $\beta_{j}$ are algebraic.

a) According to the six exponentials Theorem, if $\ell \geq 4$, then $\Gamma$ is dense in $\left(\mathbb{R}_{+}^{\times}\right)^{2}$.

For instance if $\ell=4$ and if the eight numbers $\alpha_{j}, \beta_{j}$ are multiplicatively independent, the group $\Gamma$ whose rank is 4 is dense in $\left(\mathbb{R}_{+}^{\times}\right)^{2}$.

b) If we take for granted the four exponentials Conjecture (see section $2 \mathrm{a}$ ), $\Gamma$ is dense in $\left(\mathbb{R}_{+}^{\times}\right)^{2}$ as soon as $\ell \geq 3$.

\footnotetext{
1 According to his own taste, the reader will find a reference either in N. Bourbaki, Eléments de Mathématique, Topologie Générale, Herman 1974, Chap. VII, § 1, N¹, Prop. 2; or else in G.H. Hardy and A.M. Wright, An Introduction to the Theory of Numbers, Oxford Sci. Publ., 1938, Chap. XXIII.
} 
For instance when $\ell=3$ and when the six numbers $\alpha_{j}, \beta_{j}$ are multiplicatively independent, then we expect $\Gamma$ to be dense in $\left(\mathbb{R}_{+}^{\times}\right)^{2}$.

Example: the field $\mathbb{Q}(\sqrt{2})$.

From the six exponentials Theorem follows that the subgroup of $\left(\mathbb{R}^{\times}\right)^{2}$, of rank 4 , which is generated by the images of

$$
2 \sqrt{2}-1, \quad-3 \sqrt{2}-1, \quad 4 \sqrt{2}-1, \quad 6 \sqrt{2}-1,
$$

under the canonical embedding of the real quadratic field $\mathbb{Q}(\sqrt{2})$, is dense in $\left(\mathbb{R}^{\times}\right)^{2}$. If we knew the four exponentials Conjecture we could omit $6 \sqrt{2}-1$ and get a dense subgroup of rank 3 .

a2) Consequences of the six exponentials Theorem: complex case

Consider $\ell$ multiplicatively independent complex numbers $\gamma_{1}, \ldots, \gamma_{\ell}$. The subgroup $\Gamma$ of $\mathbb{C}^{\times}$generated by $\gamma_{1}, \ldots, \gamma_{\ell}$ is dense if and only if for all $s \in \mathbb{Z}^{\ell+1} \backslash\{0\}$, the matrix with three rows and $\ell+1$ columns

$$
\left(\begin{array}{cccc}
0 & \log \left|\gamma_{1}\right| & \cdots & \log \left|\gamma_{\ell}\right| \\
2 \pi i & \log \left(\gamma_{1} / \bar{\gamma}_{1}\right) & \cdots & \log \left(\gamma_{\ell} / \bar{\gamma}_{\ell}\right) \\
s_{0} & s_{1} & \cdots & s_{\ell}
\end{array}\right)
$$

has rank 3 (this condition clearly does not depend on the choice of the logarithms $\left.\log \left(\gamma_{j} / \bar{\gamma}_{j}\right)\right)$. A first necessary condition is that at least two of the numbers $\left|\gamma_{1}\right|, \ldots,\left|\gamma_{\ell}\right|$ are multiplicatively independent; a second necessary condition is that the numbers $\gamma_{1} /\left|\gamma_{1}\right|, \ldots, \gamma_{\ell} /\left|\gamma_{\ell}\right|$ are not all roots of unity. These two conditions mean that the projection of $\Gamma$ on each of the two factors $\mathbb{R}_{+}^{\times}$and $\mathbb{R} / \mathbb{Z}$ which arises from $z \mapsto(|z|, z /|z|)$, has a dense image. We assume that these conditions are satisfied, and furthermore that the $\ell$ complex numbers $\gamma_{j}$ are algebraic. According to the four exponentials Conjecture, $\Gamma$ should be dense in $\mathbb{C}^{\times}$without any further assumption. On the other hand, if we use the six exponentials Theorem, assuming that three at least of the numbers $\left|\gamma_{1}\right|, \ldots,\left|\gamma_{\ell}\right|$ are multiplicatively independent, we deduce that $\Gamma$ is dense in $\mathbb{C}^{\times}$.

Example: the field $\mathbb{Q}(i)$.

The subgroup of rank 3 in $\mathbb{C}^{\times}$which is generated by

$$
2+i, \quad 2+3 i, \quad 4+i,
$$

is dense $\mathbb{C}^{\times}$. If the four exponential Conjecture holds, then for instance the subgroup of rank 2

$$
\left\{(2+i)^{s} 2^{t} ;(s, t) \in \mathbb{Z}^{2}\right\}
$$


generated by 2 and $2+i$ is dense in $\mathbb{C}^{\times}$; a proof of this result would follow from a special case of the four exponentials Conjecture: it would suffice to show that the three numbers

$$
\log 2, \quad \log 5, \quad \frac{\log 2}{2 \pi i} \cdot \log \left(\frac{3+4 i}{5}\right)
$$

are $\mathbb{Q}$-linearly independent, which means that for each $(\lambda, \mu) \in \mathbb{Q}^{2}$, the determinant

$$
\operatorname{det}\left(\begin{array}{cc}
\log 5 & \log 2 \\
\log \left(\frac{3+4 i}{5}\right)+2 \lambda \pi i & 2 \mu \pi i
\end{array}\right)
$$

does not vanish. This is not yet known.

b) Product of the multiplicative group with an elliptic curve

Let $\wp$ be a Weierstrass elliptic function with real algebraic invariants $g_{2}, g_{3}$ :

$$
\wp^{\prime 2}=4 \wp^{3}-g_{2} \wp-g_{3}
$$

the set of real points on the corresponding elliptic curve $E$, namely

$$
E(\mathbb{R})=\left\{(x: y: t) \in \mathbb{P}_{2}(\mathbb{R}) ; y^{2} t=4 x^{3}-g_{2} x t^{2}-g_{3} t^{3}\right\},
$$

has one or two connected components, according as the polynomial $4 X^{3}-g_{2} X-g_{3}$ has one or three real roots; we denote by $E(\mathbb{R})^{0}$ the connected component of $E(\mathbb{R})$ which contains the origin $(0: 1: 0)$; hence $E(\mathbb{R})^{0}$ is a subgroup of $E(\mathbb{R})$ of index 1 or 2 . For simplicity of notation, when $\omega$ is a pole of $\wp$, then $\left(\wp(\omega): \wp^{\prime}(\omega): 1\right)$ means $(0: 1: 0)$. With this convention the map $\exp _{E}: u \mapsto\left(\wp(u): \wp^{\prime}(u): 1\right)$ is a surjective homomorphism from $\mathbb{R}$ onto $E(\mathbb{R})^{0}$ whose kernel is of the form $\mathbb{Z} \omega$, where $\omega$ is a fundamental real period of $\wp$. A point $\gamma=\exp _{E}(u)=\left(\wp(u): \wp^{\prime}(u): 1\right) \in E(\mathbb{R})^{0}$ is a torsion point if and only if the two number $u$ and $\omega$ are linearly dependent over $\mathbb{Q}$. More generally, when $u_{1}, \ldots, u_{\ell}$ are real numbers, the rank over $\mathbb{Z}$ of the subgroup $\Gamma$ generated by the $\ell$ points $\gamma_{j}=\left(\wp\left(u_{j}\right): \wp^{\prime}\left(u_{j}\right): 1\right),(1 \leq j \leq \ell)$ in $E(\mathbb{R})^{0}$ is related to the rank of the subgroup $Y$ of $\mathbb{R}$ generated by the $\ell+1$ real numbers $u_{1}, \ldots, u_{\ell}, \omega$ by $\operatorname{rank}_{\mathbb{Z}} \Gamma=\operatorname{rank}_{\mathbb{Z}} Y-1$.

Recall (Kronecker's Theorem again) that a subgroup of rank $\geq 1$ in $\mathbb{R} / \mathbb{Z}$ is dense. We deduce:

Let $E$ be an elliptic curve which is defined over $\mathbb{R}$ and let $\gamma$ be a point of infinite order on $E(\mathbb{R})^{0}$; then the subgroup $\mathbb{Z} \gamma$ is dense for the real topology in $E(\mathbb{R})^{0}$.

The next density result deals with the product of the multiplicative group of non-zero real numbers with $E(\mathbb{R})$; it will be proved as a consequence of Corollary 2.5. 
Corollary 5. - Let $\alpha_{1}, \ldots, \alpha_{\ell}$ be multiplicatively independent positive real algebraic numbers; let $\gamma_{1}, \ldots, \gamma_{\ell}$ be points on $E(\overline{\mathbb{Q}}) \cap E(\mathbb{R})^{0}$, which are not all torsion points. Denote by $\Gamma$ the subgroup of $\mathbb{R}_{+}^{\times} \times E(\mathbb{R})^{0}$ which is spanned by the $\ell$ points $\left(\alpha_{j}, \gamma_{j}\right)$, $(1 \leq j \leq \ell)$.

a) Assume that $\ell \geq 4$. Then $\Gamma$ is dense in $\mathbb{R}_{+}^{\times} \times E(\mathbb{R})^{0}$.

b) According to Ramachandra's $\delta$-Conjecture $\delta\left(e^{z}, \wp(t z)\right) \leq 1$, the same conclusion should hold as soon as $\ell \geq 2$.

Proof. The exponential map

$$
\begin{aligned}
\mathbb{R}^{2} & \longrightarrow \mathbb{R}_{+}^{\times} \times E(\mathbb{R})^{0} \\
\left(x_{1}, x_{2}\right) & \longmapsto\left(e^{x_{1}} ;\left(\wp\left(x_{2}\right): \wp^{\prime}\left(x_{2}\right): 1\right)\right)
\end{aligned}
$$

is a topological surjective homomorphism with kernel $\mathbb{Z}(0, \omega)$ for some $\omega \in \mathbb{R}^{\times}$; define $y_{0}=(0, \omega)$ and $y_{j}=\left(\log \alpha_{j}, u_{j}\right),(1 \leq j \leq \ell)$, where $u_{j} \in \mathbb{R}$ is such that $\gamma_{j}=\left(\wp\left(u_{j}\right): \wp^{\prime}\left(u_{j}\right): 1\right)$. Now the goal is to prove that the subgroup $Y=\mathbb{Z} y_{0}+\mathbb{Z} y_{1}+\cdots+\mathbb{Z} y_{\ell}$ is dense in $\mathbb{R}^{2}$. Let $\varphi: \mathbb{R}^{2} \rightarrow \mathbb{R}$ be a linear form which satisfies $\varphi(Y) \subset \mathbb{Z}$. According to Kronecker's above mentioned Theorem (see footnote $\left({ }^{1}\right)$ ), we only need to prove $\varphi=0$. Write $\varphi\left(y_{j}\right)=s_{j}$ with $s_{j} \in \mathbb{Z},(0 \leq j \leq \ell)$; then the matrix

$$
\left(\begin{array}{cccc}
0 & \log \alpha_{1} & \cdots & \log \alpha_{\ell} \\
\omega & u_{1} & \cdots & u_{\ell} \\
s_{0} & s_{1} & \cdots & s_{\ell}
\end{array}\right)
$$

has rank $<3$; it follows that the rank of the matrix

$$
\left(\begin{array}{ccc}
s_{0} \log \alpha_{1} & \cdots & s_{0} \log \alpha_{\ell} \\
s_{0} u_{1}-s_{1} \omega & \cdots & s_{0} u_{\ell}-s_{\ell} \omega
\end{array}\right)
$$

is $<2$. Since $u_{1}, \ldots, u_{\ell}$ are not all torsion points and $\log \alpha_{1}, \ldots, \log \alpha_{\ell}$ are linearly independent over $\mathbb{Q}$, it follows from Corollary 2.5 that $s_{0}=0$; using once more the linear independence of the $\log \alpha$ 's, we deduce $s_{1}=\cdots=s_{\ell}=0$, and $\varphi=0$.

Remark: Ramachandra's $\delta$-Conjecture $\delta\left(e^{z}, \wp(t z)\right) \leq 1$ implies the following: Let $\log \alpha_{1}$ and $\log \alpha_{2}$ be two $\mathbb{Q}$-linearly independent logarithms of algebraic numbers. Let $E$ be an elliptic curve which is defined over the field $\overline{\mathbb{Q}}$ of algebraic numbers, $\omega$ be a non-zero period of $\exp _{E}$ and $u \in \mathbb{C}$ be an elliptic logarithm of a point of infinite order in $E(\overline{\mathbb{Q}})$. Then the three numbers

$$
\frac{\log \alpha_{1}}{\log \alpha_{2}}, \frac{u}{\omega}, 1
$$


are linearly independent over $\mathbb{Q}$.

Indeed, this means, for each $(\lambda, \mu) \in \mathbb{Q}^{2}$,

$$
\operatorname{det}\left(\begin{array}{cc}
\log \alpha_{1} & \log \alpha_{2} \\
u+\lambda \omega & \mu \omega
\end{array}\right) \neq 0
$$

c) Product of two elliptic curves

As a consequence of Corollary 2.6 we have a density result for the product of two elliptic curves over the real number field.

Corollary 6. - Let $E$ and $E^{*}$ be two Weierstrass elliptic curves with real algebraic invariants $g_{2}, g_{3}$ and $g_{2}^{*}, g_{3}^{*}$ respectively; assume for simplicity that there is no isogeny between them, which means that for $t \in \mathbb{C}^{\times}$, the two functions $\wp(t z)$ and $\wp^{*}(z)$ are algebraically independent. Denote by $\omega$ (resp. $\left.\omega^{*}\right)$ a non-zero real period of $\wp$ (resp. of $\left.\wp^{*}\right)$. Let $\ell$ be a positive integer and let $\gamma_{1}, \ldots, \gamma_{\ell}\left(\right.$ resp. $\left.\gamma_{1}^{*}, \ldots, \gamma_{\ell}^{*}\right)$ be elements in $E(\mathbb{R})^{0} \cap E(\overline{\mathbb{Q}})$ (resp. in $\left.E^{*}(\mathbb{R})^{0} \cap E^{*}(\overline{\mathbb{Q}})\right)$ such that

a) $\gamma_{1}, \ldots, \gamma_{\ell}$ are not all torsion points on $E(\overline{\mathbb{Q}})$;

b) $\gamma_{1}^{*}, \ldots, \gamma_{\ell}^{*}$ are not all torsion points on $E^{*}(\overline{\mathbb{Q}})$;

c) the subgroup $\Gamma$ of $E(\overline{\mathbb{Q}}) \times E^{*}(\overline{\mathbb{Q}})$ generated by the $\ell$ points $\left(\gamma_{j}, \gamma_{j}^{*}\right),(1 \leq j \leq \ell)$ has rank $\ell$.

Then

1) if $\ell \geq 3, \Gamma$ is dense in $E(\mathbb{R})^{0} \times E^{*}(\mathbb{R})^{0}$.

2) if Ramachandra's $\delta$-Conjecture $\delta\left(\wp(\omega z), \wp^{*}\left(\omega^{*} z\right)\right) \leq 1$ is true, the same conclusion holds already for $\ell \geq 1$.

Proof: We first translate the hypotheses concerning the points on the elliptic curves in terms of elliptic logarithms. Let $\omega$ (resp. $\omega^{*}$ ) be a real fundamental period of $\wp\left(\right.$ resp. of $\left.\wp^{*}\right)$; for $1 \leq j \leq \ell$, let $u_{j} \in \mathbb{R}$ satisfy $\left(\wp\left(u_{j}\right): \wp^{\prime}\left(u_{j}\right): 1\right)=\gamma_{j}$, and let $u_{j}^{*} \in \mathbb{R}$ satisfy $\left(\wp^{*}\left(u_{j}^{*}\right): \wp^{* \prime}\left(u_{j}^{*}\right): 1\right)=\gamma_{j}^{*}$. Now $u_{1}, \ldots, u_{\ell}\left(\operatorname{resp} . u_{1}^{*}, \ldots, u_{\ell}^{*}\right)$ are pseudo-algebraic points of $\wp$ (resp. of $\left.\wp^{*}\right)$, such that

a) two at least of the $\ell+1$ numbers $u_{1}, \ldots, u_{\ell}, \omega$ are linearly independent over $\mathbb{Q}$,

b) two at least of the $\ell+1$ numbers $u_{1}^{*}, \ldots, u_{\ell}^{*}, \omega^{*}$ are linearly independent over $\mathbb{Q}$,

c) the $\ell+2$ points

$$
y_{0}=\left(\begin{array}{c}
\omega \\
0
\end{array}\right), \quad y_{0}^{*}=\left(\begin{array}{c}
0 \\
\omega^{*}
\end{array}\right), \quad y_{j}=\left(\begin{array}{c}
u_{j} \\
u_{j}^{*}
\end{array}\right), \quad(1 \leq j \leq \ell)
$$

are linearly independent over $\mathbb{Q}$. We want to prove that the subgroup of $\mathbb{R}^{2}$ of rank $\ell+2$ which is generated by $y_{0}, y_{0}^{*}, y_{1}, \ldots, y_{\ell}$ is dense in $\mathbb{R}^{2}$. Indeed, let $s_{0}, s_{0}^{*}, s_{1}, \ldots, s_{\ell}$ 
be rational integers, not all of which are zero; we shall deduce from Corollary 2.6 (with a shift of notations $\ell \mapsto \ell+1$ ) that the matrix

$$
\left(\begin{array}{ccccc}
\omega & 0 & u_{1} & \cdots & u_{\ell} \\
0 & \omega^{*} & u_{1}^{*} & \cdots & u_{\ell}^{*} \\
s_{0} & s_{0}^{*} & s_{1} & \cdots & s_{\ell}
\end{array}\right)
$$

has rank 3. If $s_{0}=0$ (resp if $s_{0}^{*}=0$ ), this follows from the hypothesis b) (resp. a)) above. Assume now $s_{0} \neq 0$ and $s_{0}^{*} \neq 0$; we want to prove that the matrix

$$
\left(\begin{array}{cccc}
-s_{0}^{*} \omega & s_{0} u_{1}-s_{1} \omega & \cdots & s_{0} u_{\ell}-s_{\ell} \omega \\
\omega^{*} & u_{1}^{*} & \cdots & u_{\ell}^{*}
\end{array}\right)
$$

has rank 2 ; in order to use part b) of Corollary 2.6, we need to check that the $\ell+1$ elements on the first row are linearly independent over $\mathbb{Q}$, and the same for the $\ell+1$ elements on the second row; if this were not the case, and if the matrix had rank $<2$, we would get a non trivial linear dependence relation between the $\ell+2$ elements

$$
\left(\begin{array}{c}
\omega \\
0
\end{array}\right), \quad\left(\begin{array}{c}
0 \\
\omega^{*}
\end{array}\right), \quad\left(\begin{array}{l}
u_{j} \\
u_{j}^{*}
\end{array}\right), \quad(1 \leq j \leq \ell),
$$

which would contradict the assumption $\operatorname{rank}_{\mathbb{Z}} \Gamma=\ell$.

Remark: Ramachandra's $\delta$-Conjecture $\delta\left(\wp(\omega z), \wp^{*}\left(\omega^{*} z\right)\right) \leq 1$ implies the following: Let $\wp\left(\right.$ resp. $\left.\wp^{*}\right)$ be a Weierstrass elliptic function with algebraic $g_{2}, g_{3}$ (resp. $\left.g_{2}^{*}, g_{3}^{*}\right)$; let $\omega\left(\right.$ resp. $\left.\omega^{*}\right)$ be a non-zero period of $\wp\left(\right.$ resp. of $\left.\wp^{*}\right)$, and $u \in \mathbb{C}\left(\right.$ resp. $\left.u^{*} \in \mathbb{C}\right)$ be a pseudo-algebraic point of $\wp$ (resp. of $\left.\wp^{*}\right)$, with $u / \omega$ and $u^{*} / \omega^{*}$ both irrational numbers; assume that the two complex functions $\wp(\omega z)$ and $\wp^{*}\left(\omega^{*} z\right)$ are algebraically independent; then the three numbers

$$
1, \frac{u}{\omega}, \frac{u^{*}}{\omega^{*}}
$$

are linearly independent over $\mathbb{Q}$.

In other words, according to this conjecture, for rational integers $s_{0}, s_{0}^{*}, s$, the determinant

$$
\operatorname{det}\left(\begin{array}{ccc}
\omega & 0 & u \\
0 & \omega^{*} & u^{*} \\
s_{0} & s_{0}^{*} & s
\end{array}\right)
$$

can vanish only for $s_{0}=s_{0}^{*}=s=0$. 
d) Complex points on an elliptic curve

Let $E$ be an elliptic curve over $\mathbb{C}$

$$
E(\mathbb{C})=\left\{(x: y: t) \in \mathbb{P}_{2}(\mathbb{C}) ; y^{2} t=4 x^{3}-g_{2} x t^{2}-g_{3} t^{3}\right\},
$$

and let $\gamma \in E(\mathbb{C})$; we ask whether the subgroup $\mathbb{Z} \gamma$ spanned by $\gamma$ is dense in the topological group $E(\mathbb{C})$.

Denote as before by $\wp$ the Weierstrass elliptic function with invariants $g_{2}$ and $g_{3}$ :

$$
\wp^{\prime 2}=4 \wp^{3}-g_{2} \wp-g_{3}
$$

by $\Omega=\mathbb{Z} \omega_{1}+\mathbb{Z} \omega_{2}$ the lattice of periods of $\wp$, by $\bar{\Omega}=\mathbb{Z} \bar{\omega}_{1}+\mathbb{Z} \bar{\omega}_{2}$ the complex conjugate lattice and by $\bar{E}=\mathbb{C} / \bar{\Omega}$ the Weierstrass elliptic curve with invariants $\bar{g}_{2}$ and $\bar{g}_{3}$; select $u=x_{1} \omega_{1}+x_{2} \omega_{2} \in \mathbb{C}$ (with real $x_{1}$ and $\left.x_{2}\right)$ such that $\gamma=\left(\wp(u): \wp^{\prime}(u): 1\right.$ ). The three conditions

(i) $\gamma$ is not a torsion point;

(ii) the three numbers $u, \omega_{1}, \omega_{2}$ are $\mathbb{Q}$-linearly independent;

(iii) $\left(x_{1}, x_{2}\right) \notin \mathbb{Q}^{2}$

are equivalent.

On the other hand the three following conditions are also equivalent:

$(j) \mathbb{Z} \gamma$ is dense in the topological group $E(\mathbb{C})$;

$(j j)$ the three numbers $1, x_{1}, x_{2}$ are $\mathbb{Q}$-linearly independent over $\mathbb{Q}$;

$(j j j)$ if $\omega \in \Omega$ is any non-zero period of $\wp$ and $n \geq 1$ any positive integer, then $n \gamma$ does not belong to the 1-parameter subgroup $\left\{\left(\wp(t \omega): \wp^{\prime}(t \omega): 1\right) ; t \in \mathbb{R}\right\}$ of $E(\mathbb{C})$.

Of course conditions $(j),(j j)$ et $(j j j)$ imply conditions $(i)$, (ii) et $(i i i)$; clearly the converse does not hold without any further assumption. Let us assume that $g_{2}$ and $g_{3}$ are algebraic, as well as $\wp(u)$ and $\wp^{\prime}(u)$.

The curve $E$ is defined over $\mathbb{R}$ if and only if there exists $\theta \in \mathbb{C}^{\times}$such that $\theta \Omega=\overline{\theta \Omega}$; the set

$$
E(E)=\left\{\theta \in \mathbb{C}^{\times} ; \operatorname{rank}_{\mathbb{Z}}(\theta \Omega \cap \overline{\theta \Omega})=2\right\} ;
$$

is empty if and only if the two curves $E$ and $\bar{E}$ are not isogeneous. We start with the easiest case:

Corollary 7. - Let $E$ be an elliptic curve which is defined over the field $\overline{\mathbb{Q}}$ of algebraic numbers and is not isogeneous to its complex conjugate.

1) Any subgroup of $E(\overline{\mathbb{Q}})$ of rank $\geq 3$ is dense in $E(\mathbb{C})$ for the complex topology.

2) If Ramachandra's $\delta$-Conjecture $\delta\left(\wp(\omega z), \wp^{*}\left(\omega^{*} z\right)\right) \leq 1$ is true, any element in $E(\overline{\mathbb{Q}})$ which is not a torsion point spans a dense subgroup of $E(\mathbb{C})$. 
Proof. The proof is the same as for Corollary 3.2; also, Corollary 3.3 will follow from Corollary 3.5 below.

Before we study the general case, we prove the following auxiliary lemma.

Lemma 1. - Let $\Omega=\mathbb{Z} \omega_{1}+\mathbb{Z} \omega_{2}$ be a lattice in $\mathbb{C}$; let $\theta \in \mathbb{C}^{\times}$be such that $\theta \Omega \cap \overline{\theta \Omega}$ is a subgroup of finite index in $\theta \Omega$. Let $Y$ be a finitely generated subgroup of $\mathbb{C}$. Define two subgroups of $\mathbb{C}$ by

$$
Y_{\theta}=\{\theta y-\overline{\theta y} ; y \in Y\}
$$

and

$$
\widetilde{\Omega}_{\theta}=\left\{\theta \omega-\overline{\theta \omega^{\prime}} ;\left(\omega, \omega^{\prime}\right) \in \Omega \times \Omega\right\} \subset \mathbb{C} .
$$

If $Y_{\theta} \cap \widetilde{\Omega}_{\theta}$ is a subgroup of finite index in $Y_{\theta}$, then $Y$ is not dense in $\mathbb{C}$.

Proof: a) From the hypotheses we deduce that there exists a positive integer $m$ such that $m \theta \omega_{1} \in \overline{\theta \Omega}$ and $m \theta \omega_{2} \in \overline{\theta \Omega}$. Define $a, b, c, d$ is $\mathbb{Z}$ by

$$
m \theta \omega_{1}=a \overline{\theta \omega}_{1}+b \overline{\theta \omega}_{2} \quad \text { and } \quad m \theta \omega_{2}=c \overline{\theta \omega}_{1}+d{\overline{\theta \omega_{2}}}
$$

We show the relations

$$
a+d=0 \quad \text { and } \quad m^{2}+a d-b c=0 .
$$

Using complex conjugation, we get

$$
m^{2} \overline{\theta \omega_{1}}=a m \theta \omega_{1}+b m \theta \omega_{2}=a\left(a \overline{\theta \omega}_{1}+b \overline{\theta \omega}_{2}\right)+b\left(c \overline{\theta \omega}_{1}+d \overline{\theta \omega}_{2}\right)
$$

and

$$
m^{2} \overline{\theta \omega_{2}}=c m \theta \omega_{1}+d m \theta \omega_{2}=c\left(a \overline{\theta \omega}_{1}+b \overline{\theta \omega}_{2}\right)+d\left(c \overline{\theta \omega}_{1}+d \overline{\theta \omega}_{2}\right)
$$

hence

$$
m^{2}=a^{2}+b c, \quad(a+d) b=0, \quad m^{2}=d^{2}+b c, \quad(a+d) c=0 .
$$

The solution $a=d$ and $b=c=0$ is not possible because $\omega_{2} / \omega_{1} \notin \mathbb{R}$.

b) We show that there exist $\omega_{0}$ and $\omega_{0}^{\prime}$ which generate a subgroup of finite index in $\Omega$ such that $\theta \omega_{0} \in \mathbb{R}$ and $\theta \omega_{0}^{\prime} \in i \mathbb{R}$.

We want to find $\lambda, \mu$ in $\mathbb{Z}$ such that $\omega_{0}=\lambda \omega_{1}+\mu \omega_{2}$ satisfies $\theta \omega_{0}=\overline{\theta \omega_{0}}$ : we need to solve the system

$$
\begin{aligned}
& (a-m) \lambda+c \mu=0 \\
& b \lambda+(d-m) \mu=0
\end{aligned}
$$

whose determinant $a d-b c-m(a+d)+m^{2}$ vanishes; hence there is a non trivial solution, which means that $\theta \Omega \cap \mathbb{R}$ is a $\mathbb{Z}$-module of rank 1 . Similarly, since $a d-b c+m(a+d)+m^{2}=0$, the system 


$$
\begin{aligned}
& (a+m) \lambda^{\prime}+c \mu^{\prime}=0 \\
& b \lambda^{\prime}+(d+m) \mu^{\prime}=0
\end{aligned}
$$

has a non trivial solution $\left(\lambda^{\prime}, \mu^{\prime}\right) \in \mathbb{Z}^{2}$, and $\theta \Omega \cap i \mathbb{R}$ is generated by a non-zero element $\omega_{0}^{\prime}=\lambda^{\prime} \omega_{1}+\mu^{\prime} \omega_{2}$.

c) For each $v \in \widetilde{\Omega}_{\theta} \cap i \mathbb{R}$ we show that there exist a positive integer $k$ and an element $\omega \in \Omega$ such that

$$
k v=\theta \omega-\overline{\theta \omega} .
$$

Since $v \in \widetilde{\Omega}_{\theta}$ there exist $\omega \in \Omega$ and $\omega^{\prime} \in \Omega$ such that $v=\theta \omega-\overline{\theta \omega^{\prime}}$. It follows from the previous result in b) that there exist integers $h, a, b, c, d$ with $h \geq 1$ such that

$$
h \omega=a \omega_{0}+b \omega_{0}^{\prime} \quad \text { and } \quad h \omega^{\prime}=c \omega_{0}+d \omega_{0}^{\prime} .
$$

We deduce

$$
h v=(a-c) \theta \omega_{0}+(b+d) \theta \omega_{0}^{\prime} .
$$

From the hypothesis $\bar{v}=-v$ we conclude $a=c$, and the result follows with $k=2 h$ and $\omega=(b+d) \omega_{0}^{\prime}$.

d) Define a linear form $\varphi: \mathbb{C} \rightarrow \mathbb{R}$ of $\mathbb{R}$-vector spaces by

$$
\varphi\left(x_{1} \omega_{1}+x_{2} \omega_{2}\right)=\mu x_{1}-\lambda x_{2},
$$

where $(\lambda, \mu)$ satisfies (as before) $\omega_{0}=\lambda \omega_{1}+\mu \omega_{2} \in \Omega \cap \mathbb{R}$. If $Y_{\theta} \cap \widetilde{\Omega}_{\theta}$ is a subgroup of finite index in $Y_{\theta}$, then we shall deduce $\varphi(Y) \subset \mathbb{Q}$ (from which it follows that $Y$ is not dense in $\mathbb{C}$ ).

For the proof, we take $y=x_{1} \omega_{1}+x_{2} \omega_{2} \in Y$ with $\left(x_{1}, x_{2}\right) \in \mathbb{R}^{2}$. Let $m$ be a positive integer such that $m(\theta y-\overline{\theta y}) \in \widetilde{\Omega}_{\theta}$; it follows from c) that there exist $k \geq 1$ and $\omega \in \Omega$ with

$$
m k(\theta y-\overline{\theta y})=\theta \omega-\overline{\theta \omega}
$$

and therefore $m k \theta(y-\omega) \in \mathbb{R}$. Put $n=m k$ and write $\omega=a \omega_{1}+b \omega_{2}$ :

$$
\left(n x_{1}-a\right) \theta \omega_{1}+\left(n x_{2}-b\right) \theta \omega_{2} \in \mathbb{R} ;
$$

however $\theta \omega_{1}$ and $\theta \omega_{2}$ are linearly independent over $\mathbb{R}$ and satisfy $\lambda \theta \omega_{1}+\mu \theta \omega_{2} \in \mathbb{R}$. We deduce $\lambda\left(n x_{2}-b\right)=\mu\left(n x_{1}-a\right)$, which completes the proof.

We can now state and prove the following result:

Corollary 8. - Let $E=\mathbb{C} / \Omega$ be a Weierstrass elliptic curve with algebraic $g_{2}, g_{3}$. Define

$$
E(E)=\left\{\theta \in \mathbb{C}^{\times} ; \operatorname{rank}_{\mathbb{Z}}(\theta \Omega \cap \overline{\theta \Omega})=2\right\}
$$


for each $\theta \in E(E)$, define

$$
\widetilde{\Omega}_{\theta}=\left\{\theta \omega-\overline{\theta \omega^{\prime}} ;\left(\omega, \omega^{\prime}\right) \in \Omega \times \Omega\right\} \subset \mathbb{C} .
$$

Let $\Gamma=\mathbb{Z} \gamma_{1}+\cdots+\mathbb{Z} \Gamma_{\ell}$ be a finitely generated subgroup of rank $\ell$ in $E(\overline{\mathbb{Q}})$. Define $Y \subset \mathbb{C}$ by $Y=\exp _{E}^{-1}(\Gamma)$. For each $\theta \in E(E)$, put

$$
Y_{\theta}=\{\theta y-\overline{\theta y} ; y \in Y\}
$$

Assume that for each $\theta \in E(E)$, the subgroup $Y_{\theta} \cap \widetilde{\Omega}_{\theta}$ is not of finite index in $Y_{\theta}$.

a) If $\ell \geq 3$, then $\Gamma$ is dense in $E(\mathbb{C})$.

b) If Ramachandra's $\delta$-Conjecture $\delta\left(\wp(\omega z), \wp^{*}\left(\omega^{*} z\right)\right) \leq 1$ is true, then $\Gamma$ is a dense subgroup of $E(\mathbb{C})$.

It follows that for any elliptic curve $E$ which is defined over $\overline{\mathbb{Q}}$, there exists an algebraic number field $K$ such that $E(K)$ is dense in the topological group $E(\mathbb{C})$.

Proof: A necessary and sufficient condition for $\Gamma$ to be dense in $E(\mathbb{C})$ is that $Y$ is dense in $\mathbb{C}$. For $1 \leq j \leq \ell$, let $u_{j} \in \mathbb{C}$ be an elliptic logarithm of $\gamma_{j}$; then $Y$ is dense in $\mathbb{C}$ if and only if $\mathbb{Z} \omega_{1}+\mathbb{Z} \omega_{2}+\mathbb{Z} u_{1}+\cdots+\mathbb{Z} u_{\ell}$ is dense in $\mathbb{C}$; according to Kronecker's Theorem, this is equivalent to the following assertion: for each $\left(s_{0}^{\prime}, s_{0}^{\prime \prime}, s_{1}, \ldots, s_{\ell}\right) \in \mathbb{Z}^{\ell+2} \backslash\{0\}$, the matrix

$$
\left(\begin{array}{ccccc}
\omega_{1} & \omega_{2} & u_{1} & \cdots & u_{\ell} \\
\overline{\omega_{1}} & \overline{\omega_{2}} & \overline{u_{1}} & \ldots & \overline{u_{\ell}} \\
s_{0}^{\prime} & s_{0}^{\prime \prime} & s_{1} & \cdots & s_{\ell}
\end{array}\right)
$$

has rank 3. This condition is clearly satisfied if either $s_{0}^{\prime}=0$ or $s_{0}^{\prime \prime}=0$, because we assume $\Gamma$ has rank $\ell$. If $s_{0}^{\prime} \neq 0$ and $s_{0}^{\prime} \neq 0$, we define

$$
\omega=s_{0}^{\prime} \omega_{2}-s_{0}^{\prime \prime} \omega_{1}, \quad v_{j}=s_{0}^{\prime} u_{j}-s_{j} \omega_{1}, \quad(1 \leq j \leq \ell)
$$

and we want to prove that the matrix

$$
\left(\begin{array}{cccc}
\omega & v_{1} & \cdots & v_{\ell} \\
\bar{\omega} & \overline{v_{1}} & \cdots & \overline{v_{\ell}}
\end{array}\right)
$$

has rank 2. If the two functions $\wp(\omega z)$ and $\bar{\wp}(\bar{\omega} z)$ are algebraically independent, we can apply parts b) and c) of Corollary 2.6 (with $\ell$ replaced by $\ell+1$ again). Otherwise, since the period lattices of $\wp(\omega z)$ and $\bar{\wp}(\bar{\omega} z)$ are respectively $(1 / \omega) \Omega$ and $(1 / \bar{\omega}) \bar{\Omega}$, we can use our assumption on $Y_{\theta}$ with $\theta=1 / \omega$ : firstly $\theta \Omega \cap \overline{\theta \Omega}$ is of finite index in $\theta \Omega$, secondly the numbers

$$
s_{0}^{\prime}\left(\theta u_{1}-\overline{\theta u_{1}}\right)-s_{1}\left(\theta \omega_{1}-\overline{\theta \omega_{1}}\right)
$$

do not all vanish; hence the above matrix has rank 2 . 
4. Further CONTRibutions of RAmachandRa to TRANSCEndENTAL Number THEORY

a) On the numbers $2^{\pi^{k}},(k=1,2,3, \ldots)$

From the six exponentials Theorem follows that one at least of the three numbers $2^{\pi}, 2^{\pi^{2}}$ and $2^{\pi^{3}}$ is transcendental. The result can be made effective, and a transcendence measure for at least one of these three numbers can be derived. Using a Theorem of Szemeredi, Srinivasan obtained a result which he himself states as follows: for almost all $k$, the number $2^{\pi^{k}}$ has a transcendence measure of the type

$$
\left|2^{\pi^{k}}-\alpha\right| \geq \exp \left\{-\left(\log H_{\nu}\right)^{1+\epsilon}\right\}
$$

(for any $\epsilon>0$ with respect to a sequence of heights $H_{\nu} \rightarrow \infty$, with height of $\alpha$ bounded by $\left.H_{\nu}\right)$.

Starting from such a statement, he investigated the number of algebraic numbers among the numbers $2^{\pi^{k}},(1 \leq k \leq N)$; in [53] and [54] he got the upper bound $O(\sqrt{N})$ (conjecturally, none of them is algebraic). The $O$ constant was bounded by 2 in [42] and by $\sqrt{2}$ in [4]. In fact, $O(\sqrt{N})$ can be replaced by a more explicit expression of the type $c \sqrt{N}+$ smaller order terms.

b) A note on Baker's method

Ramachandra has several contributions to Baker's theory on linear forms in logarithms and its applications; see in particular [38], [39], and [41]. A survey of this subject is given in [3]. It is fair to quote here also the important work of Shorey: [50], 51] and [52] (one of the main contributions of Ramachandra's to transcendental number theory is Shorey).

For his investigations concerning lower bounds for linear forms in logarithms of algebraic numbers, Ramachandra used Baker's method (which is a generalization of Gel'fond's solution to Hilbert's seventh problem). It turns out that a method closely related to [36] yields similar results; see [31], 64] and [27] for "usual" logarithms, and [69] for elliptic logarithms.

The paper 38] provides the first lower bound for simultaneous linear forms in logarithms. This was done at a very early stage of Baker's method, and the estimate has been superseded now, but the interest lies in the idea of improving the bound by considering several simultaneous linear forms; also the suggestion that the simultaneous result should allow one to conjecture a stronger result for a single linear form turned out to be correct. 
The subject has been developed more recently in [28], 35] and [18]. Using the same argument as in [38], one might consider that these work give partial evidence towards the Lang-Waldschmidt Conjectures (see the Introduction to Chapters X and XI of [23]).

c) An easy transcendence measure for $e$

When $\theta$ is a complex transcendental number, a transcendence measure for $\theta$ is a lower bound for $|P(\theta)|$ when $P$ is a non-zero polynomial with rational integer coefficients. Such a lower bound should depend on the degree of the polynomial $P$ as well as on the height $H(P)$ of the same (we consider the so-called "usual height", namely the maximum absolute value of the coefficients of $P$ ). The very first transcendence measure goes back to the nineteenth century: Borel gave a transcendence measure for $e$ in 1899. Later Popken (1929), Mahler (1932), Fel'dman (1963), Galochkin (1972), Cijsouw (1974), Durand (1980), Ramachandra (1987), Khassa and Srinivasan (1991) as well as other authors gave further transcendence measure for the same number $e$. We quote here the result of [19] which rests on Ramachandra's method in [40].

For every positive integer $n$ there exists a constant $H_{0}$ which depends only on $n$ such that for each positive integer $m$, each positive real number $H \geq H_{0}$ and each non-zero polynomial $P \in \mathbb{Z}[X]$ of degree $\leq n$, with at most $m$ non-zero coefficients, and with height $H(P) \leq H$,

$$
|P(e)| \geq H^{-m-\frac{c m n \log (m+1)}{\log \log H}}
$$

An interesting feature of this statement is that it takes into account the number of non-zero coefficients of the polynomial in place of the degree; such an idea appears for instance in some works dealing with complexity theory; in Diophantine approximation it occurs in papers connected with Lehmer's Conjecture; it seems it never occurred before in connection with transcendence measures.

\section{OPEN PROBLEMS}

We have already seen a few unsolved questions, notably the four exponentials Conjecture and Ramachandra's $\delta$-Conjecture. Here are further unsolved questions.

\section{a) Algebraic independence}

Assume $x_{1}, \ldots, x_{d}$ are $\mathbb{Q}$-linearly independent complex numbers, and $y_{1}, y_{2}, y_{3}$ are $\mathbb{Q}$-linearly independent complex numbers; the six exponentials Theorem shows that 
at least $d-1$ of the numbers

$$
e^{x_{i} y_{j}}, \quad(1 \leq i \leq d, j=1,2,3)
$$

are transcendental. A natural question is to ask whether at least $d-1$ of these numbers are algebraically independent. This amounts to ask if the transcendence degree of the field generated by these $3 d$ numbers is at least $d-1$. This problem was raised in [36]. The same argument was reproduced in [56] as follows: according to the four exponentials Conjecture, at least $d-1$ of the numbers

$$
e^{x_{i} y_{j}}, \quad(1 \leq i \leq d, j=1,2)
$$

are expected to be transcendental; is-it true that at least $d-1$ of these numbers are algebraically independent?

Surprisingly enough, the answer to these questions is no! For instance take $y_{1}=1$, $y_{2}=\beta, y_{3}=\beta^{2}$, where $\beta$ is cubic (resp. $y_{1}=1, y_{2}=\beta$, where $\beta$ is quadratic) if one wishes to use the six exponentials Theorem (resp. the four exponentials Conjecture), and choose

$$
d=3 k, \quad\left\{x_{1}, \ldots, x_{d}\right\}=\left\{\log \alpha_{j}, \beta \log \alpha_{j}, \beta^{2} \log \alpha_{j} ; \quad(1 \leq j \leq k)\right\}
$$

(resp.

$$
\left.d=2 k, \quad\left\{x_{1}, \ldots, x_{d}\right\}=\left\{\log \alpha_{j}, \beta \log \alpha_{j} ;(1 \leq j \leq k)\right\}\right),
$$

where $\alpha_{1}, \ldots, \alpha_{k}$ are multiplicatively independent algebraic numbers; the transcendence degree is at most $2 k=2 d / 3$ (resp. $k=d / 2$ ).

One way of repairing the conjecture (see [58] conjecture 7.5.5 and exercice 7.5.b) is to assume that $y_{1}, y_{2}$ are linearly independent over the field of algebraic numbers. A better view of looking at this kind of problem from a conjectural point of view is to consider Schanuel's Conjecture [22] Chapter 3 p. 30.

The first results of algebraic independence in this direction are due to Gel'fond [15]: if $\ell d \geq 2(\ell+d)$, then the transcendence degree $t$ of the field generated by the de numbers

$$
e^{x_{i} y_{j}}, \quad(1 \leq i \leq d, 1 \leq j \leq \ell)
$$

is at least 2. Gel'fond's statement involved a so-called "technical hypothesis" (measure of linear independence for the $x_{i}$ 's, and also for the $y_{j}$ 's), which was removed later by Tijdeman (see [58] Chapitre 7).

In the early 70's, W.D.Brownawell and A.A.Smelev succeeded to prove $t \geq 3$ under suitable assumptions; in 1974, Chudnovsky obtained $2^{t} \geq \ell d /(\ell+d)$; references are given in [9]. P.Philippon reached the estimate $t+1 \geq \ell d /(\ell+d)$ in [33]; this was improved by G. Diaz in [10] as $t \geq[\ell d /(\ell+d)]$ provided that $\ell d>\ell+d$ (without 
the proviso, the four exponentials Conjecture would follow!). An interesting fact is that all these results on "large transcendence degree" always involve a "technical hypothesis"; it is an open problem to remove it.

The above mentioned theorems of algebraic independence deal with the usual exponential function; here again, extensions can be given to commutative algebraic groups; we only quote [8] and [61] which include extensions to results of algebraic independence of Ramachandra's transcendence results concerning the exponential and elliptic functions.

\section{b) Schneider's second problem in [49]}

Ramachandra's method might be the right way towards a solution of the second of Schneider's eight problems in [49]: to prove Schneider's Theorem on the transcendence of $j(\tau)$ for $\tau$ an algebraic number in the upper half plane by means of the modular function (and not by mean of elliptic functions). The best known results in this direction are in [55].

c) Linear independence of elliptic logarithms in the non CM case by Schneider's method

We already quoted the paper [69] where lower bounds for linear forms in elliptic logarithms are provided, by means of a method which is closely related to [36]; as a matter of fact, an assumption is needed: namely one assumes that the elliptic curves has non trivial endomorphisms (CM case). It is not known how to extend the method to the non-CM case.

\section{d) Effective results}

Ramachandra was concerned (see top of p. 67 in [36]) by the fact that his simplification of Schneider's method might be at the cost of making the proof ineffective in questions of transcendence measures. A quarter of a century later, we know that effectivity is not lost by avoiding derivatives. The earliest work in this direction is [Srinivasan 1974]; further developments have already been quoted ([31] and [64] for instance). However it is clear that a lot of work is still to be performed in this direction, and plenty of results are waiting to be unraveled by future generations of mathematicians.

\section{Recent references}

We give only a few references to papers or books which have been published during the last 10 years: [11], [32], [66], [13], [67], [44], [12] and [68]. They contain further references to related works. 


\section{REFERENCES}

[1] Alaoglu, L. and Erdős, P., On highly composite and similar numbers, Trans. Amer. Math. Soc., 56 (1944), 448-469.

[2] Baker, A., Transcendental Number Theory; Cambridge Univ. Press, 1975; 2nd ed. 1979.

[3] Baker, A., The theory of linear forms in logarithms; Chap.1 of: Transcendence Theory: Advances and Applications, Proc. Conf. Cambridge 1976, ed. A.Baker and D.W.Masser, Academic Press (1977), 1-27.

[4] Balasubramanian, R. and Ramachandra, K., Transcendental numbers and a lemma in combinatorics; Proc. Sem. Combinatorics and Applications, Indian Stat. Inst., (1982), 57-59.

[5] Bertrand, D. and Masser, D. W., Linear forms in elliptic integrals; Invent. Math., 58 (1980), 283-288.

[6] Bombieri, E., Algebraic values of meromorphic maps; Invent. Math., 10 (1970), 267-287; 11 (1970), 163-166.

[7] Bombieri, E. and Lang, S., Analytic subgroups of group varieties; Invent. Math., 11 (1970), $1-14$.

[8] Brownawell, W. D. and Kubota, T., The algebraic independence of Weierstrass functions and some related numbers; Acta Arith., 33 (1977), 111-149.

[9] Chudnovsky, G. V., Contributions to the theory of transcendental numbers; Mathematical Surveys and Monographs 19, Amer. Math. Soc., 1984.

[10] Diaz, G., Grands degrés de transcendance pour des familles d'exponentielles en plusieurs variables; J. Number Theory, 31 (1989), 1-23.

[11] Diaz, G., La conjecture des quatre exponentielles et les conjectures de D. Bertrand sur la fonction modulaire; J. Théor. Nombres Bordeaux 9 (1997), no. 1, p. 229-245.

[12] Diaz, G., Utilisation de la conjugaison complexe dans létude de la transcendance de valeurs de la fonction exponentielle; J. Théor. Nombres Bordeaux 16 (2004), no. 3, 535-553.

[13] Fel'dman, N. I. and Nesterenko, Yu. V., Number theory. IV. Transcendental Numbers; Encyclopaedia of Mathematical Sciences, 44. Springer-Verlag, Berlin, 1998.

[14] Gel'fond, A. O., On Hilbert's seventh problem; Dokl. Akad. Nauk SSSR, 2 (1934), 1-3 (in Russian) and 4-6 (in French); Sur le septième problème de Hilbert; Izv. Akad. Nauk SSSR, 7 (1934), 623-630.

[15] Gel'fond, A. O., Transcendental Number Theory; Moscow, 1952; English transl. Dover Publ., N.Y., 1960.

[16] Gramain, F. and Mignotte, M., Fonctions entières arithmétiques; Approximations diophantiennes et nombres transcendants, Luminy 1982, Progress in Math. 31, Birkhäuser 1983, 99 124.

[17] Gramain, F., Mignotte, M. and Waldschmidt, M., Valeurs algébriques de fonctions analytiques; Acta Arith., 47 (1986), 97-121.

[18] Hirata, N., Approximations simultanées sur les groupes algébriques commutatifs; Compositio Math., 86 (1993), 69-96.

[19] Khassa, D. S. and Srinivasan, S., A transcendence measure for e; J. Indian Math. Soc., 56 (1991), 145-152.

[20] Lang, S., Nombres transcendants, Sém. Bourbaki 18ème année (1965/66), № 305.

[21] Lang, S., Algebraic values of meromorphic functions, II, Topology, 5 (1966), 363-370. 
[22] Lang, S., Introduction to Transcendental Numbers; Addison-Wesley 1966.

[23] Lang, S., Elliptic Curves Diophantine Analysis; Grund. der math. Wiss., 231, Springer Verlag (1978).

[24] Laurent, M., Sur quelques résultats récents de transcendance; Journées arithmétiques Luminy 1989, Astérisque, 198-200 (1991), 209-230.

[25] Laurent, M., Hauteurs de matrices d'interpolation; Approximations Diophantiennes et Nombres Transcendants, Luminy 1990, éd. P. Philippon, de Gruyter 1992, 215-238.

[26] Laurent, M., Linear forms in two logarithms and interpolation determinants. Acta Arith. 66 (1994), no. 2, 181-199.

[27] Laurent, M., Mignotte, M. and Nesterenko, Yu. V., Formes linéaires en deux logarithmes et déterminants d'interpolation. J. Number Theory 55 (1995), no. 2, 285-321.

[28] Loxton, J., Some problems involving powers of integers; Acta Arith., 46 (1986), 113-123.

[29] Mahler, K., Lectures on Transcendental Numbers; Lecture Notes in Math., 546, SpringerVerlag 1976.

[30] Mignotte, M. and Waldschmidt, M., Approximation des valeurs de fonctions transcendantes Koninkl. Nederl. Akad. van Wet. Proc., Ser.A, 78 (=Indag. Math., 37), (1975), 213-223.

[31] Mignotte, M. and Waldschmidt, M., Linear forms in two logarithms and Schneider's method Math. Ann., 231 (1978), 241-267.

[32] Nesterenko, Yu. V. and Philippon, P., Introduction to algebraic independence theory; Yuri V.Nesterenko and Patrice Philippon Eds, Instructional conference (CIRM Luminy 1997). Lecture Notes in Math., 1752, Springer, Berlin-New York, (2001).

[33] Philippon, P., Critères pour l'indépendance algébrique; Publ. Math. I.H.E.S., 64 (1986), $5-52$.

[34] Philippon, P., Nouveaux aspects de la transcendance, Journées Arithmétiques (Bordeaux, 1993). J. Théor. Nombres Bordeaux 7 (1995), no. 1, 191-218. See also: Transcendance sur les anneaux diophantiens; Séminaire de Théorie des Nombres de Caen 1992-93, Univ. Caen 1994.

[35] Philippon, P. and Waldschmidt, M., Formes linéaires de logarithmes simultanées sur les groupes algébriques commutatifs; Sém. Th. Nombres Paris 1986-87, Birkhäuser Verlag, Progress in Math. 75 (1989), 313-347.

[36] Ramachandra, K., Contributions to the theory of transcendental numbers (I); Acta Arith., 14 (1968), 65-72; (II), id., 73-88.

[37] Ramachandra, K., Lectures on transcendental numbers; The Ramanujan Institute, Univ. of Madras, 1969, $72 \mathrm{p}$.

[38] Ramachandra, K., A note on Baker's method; J. Austral. Math. Soc., 10 (1969), 197-203.

[39] Ramachandra, K., Application of Baker's theory to two problems considered by Erdős and Selfridge; J. Indian Math. Soc., 37 (1973), 25-34.

[40] Ramachandra, K., An easy transcendence measure for e; J. Indian Math. Soc., 51 (1987), $111-116$.

[41] Ramachandra, K. and Shorey, T. N., On gaps between numbers with a large prime factor; Acta Arith., 24 (1973), 99-111.

[42] Ramachandra, K. and Srinivasan, S., A note to a paper by Ramachandra on transcendental numbers; Hardy-Ramanujan Journal, 6 (1983), 37-44. 
[43] Roy, D., Matrices whose coefficients are linear forms in logarithms; J. Number Theory, 41 (1992), 22-47.

[44] Roy, D., An arithmetic criterion for the values of the exponential function; Acta Arith., 97 (2001), 183-194.

[45] Schneider, T., Transzendenzuntersuchungen periodischer Funktionen, (I); Transendenz von Potenzen; J. reine angew. Math., 14 (1934), 65-69.

[46] Schneider, T., Transzendenzuntersuchungen periodischer Funktionen, (II); Transzendenzeigenschaften elliptischer Funktionen; J. reine angew. Math., 14 (1934), 70-74.

[47] Schneider, T., Zur Theorie der Abelschen Funktionen und Integrale; J. reine angew. Math., 183 (1941), 110-128.

[48] Schneider, T., Ein Satz über ganzwertige Funktionen als Prinzip für Transzendenzbeweise; Math. Ann., 121 (1949), 131-140.

[49] Schneider, T., Einführung in die transzendenten Zahlen; Springer Verlag 1957; trad. franç., Introduction aux Nombres Transcendants, Paris, Gauthier-Villars.

[50] Shorey, T. N., Linear forms in the logarithms of algebraic numbers with small coefficients; J. Indian Math. Soc., 38 (1974), 271-292.

[51] Shorey, T. N., On gaps between numbers with a large prime factor, (II); Acta Arith., 25 (1974), 365-373.

[52] Shorey, T. N., On linear forms in the logarithms of algebraic numbers; Acta Arith., 30 (1976), $27-42$.

[53] Srinivasan, S., On algebraic approximations to $2^{\pi^{k}}(k=1,2,3, \ldots)$; Indian J. Pure Appl. Math., 5 (1974), 513-523.

[54] Srinivasan, S., On algebraic approximations to $2^{\pi^{k}}(k=1,2,3, \ldots)$, (II); J. Indian Math. Soc., 43 (1979), 53-60.

[55] Wakabayashi, I., Algebraic values of functions on the unit disk; Proc. Prospects of Math. Sci., World Sci. Pub., (1988), 235-266.

[56] Waldschmidt, M., Indépendance algébrique des valeurs de la fonction exponentielle; Bull. Soc. Math. France, 99 (1971), 285-304.

[57] Waldschmidt, M., Propriétés arithmétiques des valeurs de fonctions méromorphes algébriquement indépendantes; Acta Arithm., 23 (1973), 19-88.

[58] Waldschmidt, M., Nombres transcendants; Lecture Notes in Math., 402, Springer-Verlag (1974), $277 \mathrm{p}$.

[59] Waldschmidt, M., Pólya's Theorem by Schneider's method; Acta Math. Acad. Sci. Hungar., 31 (1978), 21-25.

[60] Waldschmidt, M., Fonctions entières et nombres transcendants ; 103è Cong. Nat. Soc. Savantes, Nancy 1978, Sciences, Fasc. V, 303-317.

[61] Waldschmidt, M., Algebraic independence of values of exponential and elliptic functions; J. Indian Math. Soc., 48 (1984), 215-228.

[62] Waldschmidt, M., On the transcendence methods of Gel'fond and Schneider in several variables; Chap. 24 de : New Advances in Transcendence Theory, (ed. A. Baker), Proc. Conf. Durham 1986, (ed. A.Baker), Cambridge Univ. Press (1988), 375-398. 
[63] Waldschmidt, M., Algebraic values of analytic functions; International Symposium on Algebra and Number Theory, Silivri (Istanbul), 1989; Doḡa - Tr. J. of Math., 14 (1990), 70-78. Résumé en turc : Analitic fonksiyonların cebirsel değerleri.

[64] Waldschmidt, M., Linear independence of logarithms of algebraic numbers; The Institute of Mathematical Sciences, Madras, IMSc Report No 116, (1992), 168 p.

[65] Waldschmidt, M., Densité de points rationnels sur un groupe algébrique; Experiment. Math. 3 (1994), no. 4, 329-352; Errata; ibid., 4 (1995), no. 3, 255.

[66] Waldschmidt, M., Integer valued functions on products; J. Ramanujan Math. Soc., 12 (1997), no. $1,1-24$.

[67] Waldschmidt, M., Diophantine Approximation on Linear Algebraic Groups. Transcendence Properties of the Exponential Function in Several Variables; Grundlehren der Mathematischen Wissenschaften 326. Springer-Verlag, Berlin-Heidelberg, 2000.

[68] Waldschmidt, M., Variations on the Six Exponentials Theorem; with an appendix by H. Shiga: Periods of a Kummer surface. International Conference on Algebra and Number Theory, Hyderabad 2003; Algebra and number theory, 338-355, Hindustan Book Agency, Delhi, 2005.

[69] Yu Kunrui, Linear forms in elliptic logarithms; J. Number Theory, 20 (1985), 1-69.

Université P. et M. Curie (Paris VI)

Institut de Mathématiques CNRS UMR 7586

Théorie des Nombres Case 247

175, rue du Chevaleret

F-75013 PARIS

e-mail: miw@math.jussieu.fr

URL: http://www.math.jussieu.fr/ miw/ 